59

A143 






\section{DEI LAVORI}

DEL L'

\section{ACCADEMIA AGRARIA}

\section{IDI PESARE}

\section{NELL' ULTIMO QUINQUENNIO}

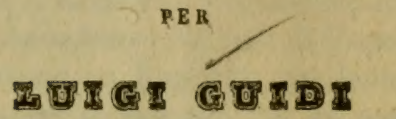

PROF. DI AGRICOLTURA TEORICO-PRATICA NELLA SCUOLA DELL' ACCADEMIA, MEMBRo DELLE SOCIETÀ METEOROLOGICA, GEOLOGICA, BOTANICA, IMPERIALE ZOOL̈OGICA DI ACCLIMATAZIONB

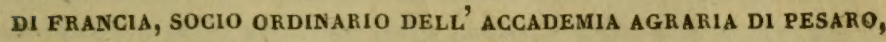
SOCIO CORRISPONDENTE DELL' ISTITUTO AGRARIO DI CAMERINO
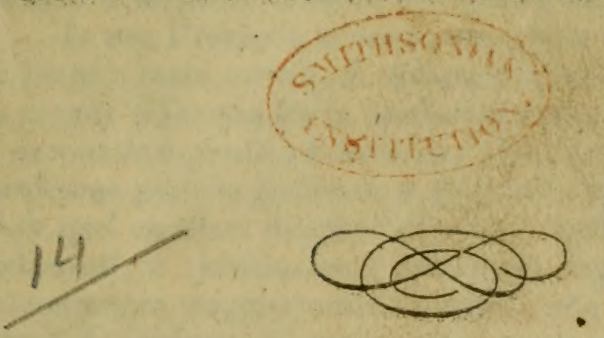

PESARO

TIPOGRAFIA NOBILI

PAR 1861 

Rapporto intorno ai Lavori dell' Accademia Agraria di Pesaro nell' ultimo quinquennio.

(Presentato al Consiglio Provinciale il 1. Febbrajo 1860.)

Illustrissimi ed Eccellentissimi Signori.

\section{Allorquando il Consiglio Provinciale nel 1855 mani-} festò il voto ehe l'Accademia Agraria di Pesaro nelle sessioni consecutive presentasse un Rapporto dei lavori compiuti nel corso dell' anno, intese principalmente ad assicurare a tutte le parti della Provincia l'operoso concorso dell' Istituto che proteggeva. Non basta in fatti che nel seno del medesimo $s^{\prime}$ istituiscano diligenti esperienze su importanti questioni di Agronomia, non basta che un attiva corrispondenza permetta di valutare nello sviluppo delle piante e nella nutrizione degli animali le influenze variabili del clima e delle stagioni.

In una Provincia sì vasta, che per la sua configurazione fa luogo a tanta varietà di colture e d' industrie, dove corre sì grande differenza fra la condizione economica dei territori, è necessario e giusto ad un tempo che i Rappresentanti della medesima possano giudicare se gli studii dell' Accademia ebbero quel carattere di geveralità che è conforme ai suoi Regolamenti, e richiamarne l' attenzione sugli argomenti che interessassero maggiormente i territorii che rappresentano. In fatti l'A 'Aricoltura all' epoca nostra non è più ristretta all' esereizio tranquillo dell' arte che in passato provvedeva facilmente ai bisogni di una popolazione meno numerosa, e più semplice. Divenendo arte più lucrosa è divenuta al tempo stesso arte più complicata, percui l' Agricoltore, che nou vuol essere sopraffatto è costretto uniformarsi al tempo in cui vive, chiedendo alle scienze i mezzi di produrre rapidamente ed a buon mercato, e trasformandosi talvolta in manifattore ed in commerciante. Particolari circostanze impedirono all' Accadenia di soddisfare uegli anui precedenti al volo del Cousi- 
glio, onde a me torna più gradito l'onore di adempiere per la prima volta, come Presidente della medesima, questo debito di ossequio e di riconoscenza, esponendo alle SS. VV. Hllme, ciò che dal nostro Istituto siasi andato operando in questi ultimi auni.

L'Accademia possiede diversi mezzi di promuovere il progresso dell' Aricoltura nella Provincia, cioè $\mathbf{I}{ }^{\circ} l$ 'istruzione, mediante la Scuola e la pubblicazione delle sue Esercitazioni. ${ }_{2} .^{\circ}$ L' esempio, mediante i metodi di coltura e il governo del bestiame nel suo Podere Modello. $3{ }^{\circ}$ Gli ajuti e gl' incoraggiamenti mediante l' esame di certe questioni speciali, e mediante i premi. Esaminaudo l' uso che l' Accademia ha fatto. di questi mezzi, le SS. VV. Illme potranno far ragione della parte che la medesima ebbe nell' evidente progresso dell' Agricoltura della nostra Provincia.

\section{Scuola}

La Scuola è altualmeute affidata al Doltor Luigi Guidr di Pesaro eletto dall' Accademia dopo la rinuncia del benemerito professore Gralvani. Il corso dura tre anni ed è pubhlico, comprendendo, conforme al Regolamento, gli Elementi delle Seienze Naturali, l'A gronomia, l'Agricoltura pratica e la Pastorizia. Un Deputato che l'Aceademia sceglie con volo. segreto fra i Membri residenti veglia al buon andamento della Scuola; alla fine di ciascun anno i Giovani debbono dar saggio del loro profitto a voce ed in iscritto inuanzi al Presidente, al Deputato e ad una Commissione scelta dall' Accademia ; e. se l' esito dell' esame fu favorevole ottengono in premio nna Medaglia d'argento o Libri d' Agricoltura. Aila fine deltriennio ricerono un diploma in cui il Presidunte ed i Censori rendono lestimonianza degli studii e del profitto che fecero, diploma che dovrebbe costituire un' efficace raceomandazione per gl' impieghi di Ministro, di Fattore ed Agente di Campagna.

L'attuale professore attenendosi strellamente at Regolamento quanto alle materie da insegnarsi, si è servito della facoltà al medesimo concedutaghi per ordinarle in modo che 


\section{-5 -}

la parte pratica e l'esame delle migliorie effeltuabili secondo la natura e lo stato economiro dei diversi territori della Provincia avessern lutta l' estensione desiderabile. Egrli espose distesamente l' ordine adottato e le ragioni che lo coudussero a preferirlo in una Menoria presentata all' Acrademia il I4 Gingno 1858 che vieue allegata in compendio sotto la lettera A affinchè le SS. VV. Illme possano far ragione dello stato della nostra Scuola , la quale è frequentata in quest' anoo, oltre ad un Uditore, che è dei buoni Possidenti della Ciltă da dieci Giovani di Pesaro e della Provincia.

L'Agricoltura però è tenuta is sì poco coutó fra uoi che, quantunque sia la prima per nou dir l'unica sorgente della uostra ricchezza, offre assai poca speranza di un collocamento couveniente a quelli che vi si dedicassero esclusivamente, per cui pochi sono disposti a consacrarvi Ire anni, studiandola i più o per ottenere il libero esercizio di alıre professioni, o per impulso dei propri padroni, o per lodevole desiderio d'apprendere, ma sempre senza quiel potente stimolo che dà la certezza di trovarvi in fine uua professione onorata e lucrosa. Siccome tale stato di cose, mutabile solo col tempo, mal si accordava con la durata del Corso piescritto dal Regolamento, il Professore sin dal i 858 (Lettera B ) tolse a fare gratuitamente anche un corso elementare da compiersi in un anno, per comodo di quelli che non potessero o non volessero dare maggior tempo allo studio dell'A gricoltura. Cinque sono vell' anno corrente i giovani iseritti per queste Lezioni, fatte sell' ora, che precede quella del Corso triennale.

\section{TPubblicazione delle Esercitagion}

Le pubblicazioni relative all' Agricoltura affrettano e completano l'opera della scuola, divulgandone gli utili insegnamenti, e gittando quei primi semi di prorresso che poi verranno fecondati per opera degli allievi della medesima. Però sifatte pubblicazioni onde produchino effetto salutare sui pratici, debbono portare l'impronta della pratica, e mostrarsi positive, semplici, veritiere. Le promesse di grossi 
guadagni chiamano un sorriso d'iucredulità sulle lahbra del Possidente avezzo a fare assegnamento su di una rendita mediocre, ma certa; e le facili asserzioni di molti scrittori di cose agrarie ginstificano la poca fiducia che vi si poue quando non riposino esclusivamente sulla osservazione e sul calcolo. Me le osservazioni non s' improvisano, ma i calcoli per essere concludenti esigono il confronto di fatti numerosi osservati in terreni e clini e stagioni diverse, l' esame diligente delle condizioni economiche del paese, e di tutte le cagioni che possouo influire sull' andamento di un ividustria tanto complessa. Ciò richiede tempo ed un concorso di circostanze che non è sempre dato oftenere, ma di cui parecchie colte persone della Provincia, ed alcuni anche fra Voi, o Signori, diedero nobile esempio. Tali furono i motivi che da tre anui fecero sospendere la pubblicazione degli Atti dell' Accademia, quantunque sia già tirata la stampa di parecchie Memorie sufficienti per comporre un fascicolo della mole ordinaria. E quelle per fermo sono di un grande interesse scientifico, ma era desiderio dell' Accademia che venissero in luce accompagnate da altre di argomento esclusivamente agrario, affinchè le Esercitazioni rispondessero esattamente al laro titolo, e potessero consultarsi con frutto dai Possidenti della Provincia. Gli studi e le esperieuze fatte dal Professore e da altri Membri dell' Accadenaia, dei quali in seguito dovrò far paiola, hauno fornito gia buon numero di dati che gg!i Autori si affretterañno di coordiuare, affiuchè nel corso dell' anno possa riprendersi la regolare pubblicazione delle nostre Effemeridi.

\section{Wodere Nodello e Sperimentale}

Il Podere Modello e Sperimentale è situato sul dor'so del vicino Monte Ardizio e comprende Tavole 227 di terreno arativo di diversa indole, che con mediocre declivio si distende sui fianchi del monte volti a mezzo-giorno, ponente e settentrione, oltre Tavole 120 di pascolo dirupatissimo nei burroni che sporgono quasi a picco sul mare. $\mathrm{Per}$ la diversità del suolo e dell' esposizione riesce adatto a quasi tatte le 
piante domestiche coltivabili velie terre di collina peponde. ranti nella Provincia, meutre per la sua giacitura e l'avara imprevidenza delle precedenti colture rimuisce tutte le difficolı̀ che si possono incontrare vell' escrcizio dell' arte.

L'Accademia, appena entrata in possessn del podere, commise al Professore un progetlo pel riordinamento completo del medesimo, ingiungendogli di esaminare minutamente i titoli di entrata e spesa per ciascuna delle migliorie proposte, distinguere le urgenti da quelle che potevano progressivameute effettuarsi, e conciliare il tutto col sistema di mezzadria praticato nella Provincia, e con gli scarsi mezzi di cui l' Accademia poteva aunualmente disporre. Il Podere era disfatto, nel senso assoluto della parola, poche viti iuvecchiate, alcuui gelsi o roveri spietatamente mutilati, non prato, nou concime, due soli eapi di grosso bestiame ed un majale su questa vasta superície esaurita da un avvicendamento secolare di grano e formentone; infine una lamiglia di contadini onesta, volenterosa, ma aggrarata dall' enorme debito di oltre Scudi I 80 coutratlo per vivere. Sarebbe lungo l' entrare nei particolari del progetto presentato dall' attuale: Professore il I 4 Luglio i 858 , e che già ebbe un principio di esecuzione, tanto più che troverà luogo uer successivi fascicoli delle Esercitazioni, ma non mi pare estraveo al fine di questa relazione esporre alle SS. VV. Illme l' idea fondamentale ed i risultati ottenuti per quella parte che venne effettuata.

Base di qualunque miglioria agraria è l'abboudanza del concime, donde seaturiscono le raccolte che servono al nostro alimento o vengono adoperate dall' industria. La regolare condotta delle acque en i lavori profondi e frequenti preparono una dimora comoda e salubre alle piante, ma ano valgoun a restiluire al terreno le materie che quelle vi assorbono durante la vegetazione e si esportano dal fondo con $\mathrm{i}$ loro prodotti. Tale verità, ripetuta al seguo da divenire volgare, trova in pratica la più viva opposizione nella nostra Provincia, dove appnnto in grazia di questa la rendita nelta della terra è inferiore a ciò che si ottieue velle Proviuteie vicine, le quali meno favorite dal clima, sono molto inferiori alla nostra nell'arte di lavorare la terra e vel governo degli alberi. Perciò chi nel territorio nostro si propune di miglio. rare un predio, si trova subito alle prese con la scarsità dei 
foraggi contro l' estensione dei quali stanno tenacissimi i preegindizj, e forse l' interesse stesso del Mezzadro. Infatti il clima della nostra Provincia non è favorevole alla produzione dei foraggi, e l'indole calcare, il poco fondo e il forte declivio delle colline coucorrono per renderne più gravi le cousegueuze. Perciò l'allevamento del bestiame, se si ecceftuino il Monte Feltro, e l'A Appennìno, dove prevale il pascolo, riesce in generale poco lucroso, e quando all' errouea determinazione della rendita della terra fatta sulla quantità dei prodolti in natura si sostituisca una regolare coutabilità, vedrassi a che realmente si riduca fra noi il guadaguo del bestiame tanto vantato. Il Professore cercò dunque per primo di accrescere la produzione dei foraggi, ma ritenendo che il bestiame costituisca per noi solamente un mezzo indiretto di accrescere la rendita del suolo mediante i lavori ed il concime; mutò contemporaneamente l'antico avvicendamento biennale adoltando una successione di colture che meglio economizzasse la debole forza produltiva di quel terreno. Ciò d'altronde diveniva una conseguenza inevitabile dell' estensione data alla coltura dei foraggi, giacchè mal si apporrebbe chi credesse l'arte agraria suscettibile di un perfezionamento importante in qualche parte, ritenendo seuza alterazioue veruna tutte le altre. Un cambiamento di qualche rilievo in quell' industria così complicata sconcerta tutto l' insieme, per cui cominciaudo la riforma di un fondo nou resta che abbandonare l'antico sistema ed accettare tutte le conseguenze del nuovo. Anche in questo però non si perdette di vista la conciliazioue dei vecchi sistemi coi nuovi, onde evitare moltissimi inconvenienti ed agevolare la via a quelli che si fossero messi ad imitarei. Il fondo venne diviso in due parti. la maggıore di Tavole 120 , come più impoverita venne destiuata ai foraggi per rientrare in progresso di tempo nell' avvicendamento ordinario; la minore di Tavole 84 veune sottoposta ad una rotazione quadriennale che si apre con una pianta sarchiata fava o formentone, ed in cui il trifoglio o le patate secondo l' indole del suolo costituiscono la coltura intermedia fra i due cereali. Nella parte destinata ai foraggi avrebbero prosperato egualmente la Medica e la Lupinella, per il sottosuolo eminentemente permeabile e calcare, e le abbondanti rugiade che vi cadono per la vicinanza del mare, ma venne 
preferita la Lupinella, come meno esigente rispetto al terreno e ai lavori. Oltrechè tale scelta venne fatta anche per uon mettere troppo in evidenza l'antagonismo creato dal sistema di Mezzadria fra il padrone ed il colono, antagonismo che nelle colture a lungo termine come la Medica si manifesta in lutta la sua gravità. Infatti a chi spettano le spese per la formazione del Medicajo? Non al padrone giacchè i produtti vanuo divisi col contadino, ma come sperare che questi alla sua volta sia contento di sostenerle quaudo scacciato dal fondo vedrebbe raccogliere da un altro il frutto delle sue fatiche? Tre anni soltanto decorsero da che il podere dell'Accademia venne ridotto a tale sistema, di cui il tempo farà ragione. Fin d'ora però si può prevedere l' esito, riflettendo che dove prina vivevano due soli bovi trovano oggi abbondante nutrimento sei capi di grosso bestiame, due vitelli, sei castrati; che in un anno si povero di foraggin si trovò modo di vendere libbre 4000 di fieno, che il colono non solo nou contrasse nuovi debiti, ma diminui l'autico di oltre scudi trenta e che tutto ciò avvenne vel volgere di tre anni quau. tunque questo sistema non potesse produrre alcon effetto nel primo anuo, e che nel secondo un Greco-Levante impetuoso mietesse in erba tutto il formentone. Infatti il vento di NordEst che spira perpeurlicolarmente al podere da due insenamenti del monte si precipita con incredibile violenza nella valle soltoposta rendendovi difficilissima la coltura arborea, e preseutando un grave ostacolo a tutte le migliorie. Il benemerito Professore Galvani nei pochi mesi trascorsi fra l'a acquisto del predio e la sua dimissione fece eseguire di fronte ai due insenameuti un argine di terra che rivestì con una folta siepe di tamarischi, piantandovi alla base una spalliera di Olmi Americani, i quali prestandosi assai bene all' intrecciamento dei rami, sostengono senza grave danno i repentini mutamenti di teniperatura. L' attuale Professore restaurò l' argine, favorì con assidue cure la laboriosa vegetazioue della spalliera, dietro la qt:ale propose una triplice cinta di alberi di alto fusto frassini o cipressi, che ponendo argine alla devastazione del vento permetterebbe di cavare qualche profitto da un suolo ingratissimo, e frattanto ridusse a pascolo la parte più esposta ai danni del vento, ritenendo essere più facile trionfare di questi ostacoli, mercè la scelta delle colture, che combattendoli direttamente. 
L' industria del bestiame nel Podere Modello venue estesa come si è detto in visla della produzione del concime, ma continuò ad essere praticata secoudo il metodo ordinario. Il miglioramento dei bestiami è opera lunga e costosa, alla quale non si deve por mano, se non dopo aver riunito tutti $i$ mezzi che possono favorirla; stalle capaci e ben ordinate, che orá mancáno affatto; varietà di foraggi; capitali per l'acquisto dei buoni riproduttori, e sopratutto cure diligentissime che il Mezzadro non sà, e più spésso non vuole adoperare. Perciò l'Accademia senza rinuzziare alla sperauza di contribuirè in seguito al progresso di questo ramo importante dell'Economia Rurale ha voluto prima che si cercasse di aumentare la forża produttiva del suolo, senza della quale tutti i teutativi per migliorare le razze riescirebbero vani.

\section{Espexienze fatte nel Podere}

Posto che il clima ed il terreuo della Provincia sieno poco favorevoli alla coltura dei foraggi importa moltissimo determinare quanto fieno possa ricavarsi da una data superficie, secondo la natura delle piante che la ricuoprono. In questo modo soltanto si arriverà a stabilire il costo di produzione del fieno e a ridurre al giusto valore l'importanza data al traffico del bestiame. Tali esperienze venuero fatte in altri paesi da Agronomi diligentissimi, e noi possiamo adottare $\mathrm{i}$ loro metodi,di ricerca, ma non i risultati variabili secondo il clima o le pratiche di coltura, le quali per differenti condizioni economiche o per il diverso genio delle popolazioni poche volte è dato imitare. Si cercò dunque di stabilire la forza produttiva della Lupinella uel Podere Modello, ritenendo che per l'indole del terreno i risultati sarebbero applicabili a quasi tutti i predi delle nostre colline. L' esperinento quantunque nou sia ancor compiuto, e convenga continuarlo sino al disfacimento del prato, è sufficiente per dimostrare quanto il prodotto della Lupinella possa aumentare mediante una diligente coltura. Infatti detratto il frutto del capitale del terreno al $5 \%$, le spese per la formazione del prato, e l'interesse di esse, si ebbe nei due anoi di raccolto l' utile 


\section{$-11-$}

netto del 55 per $\%$ sui capitali impiegati, in questa intrapresa, come all' Allegato lettera C.

II Formentone ha una grande imporlanza fra noi, dove per la quantità di materia alimentare che fornisce e il poco costo della medesima in ordine al suo valore uutritivo è divenula la coltura prediletta del Coutadino. Ma questa pianta è delle più esauriculi che si conoschino, ma ne è costosissima la coltivazione, per cui alcuni nou rifletteudo che il suo prodolto è il principale per non dir l' usico alimento del Campagnolo, proposero di baudirlo da una coltura miglioratrice. Altri invece meglio avvisati intendono soltanto a restringerne la coltivazione cercando di provvedere alla esigenze del consumo col perfezionare il metodo di coltivarlo, in modo che si aumentasse il prodotto di esso per una data superficie seuza inutile consuno delle fertilità della terra. Difficile problema, ma non iusolubile per chi rifletta a ciò che l'arte ha potuto per aumentare la forza produttiva di certe piante, e trionfare degli ostacoli della natura. Quattro sistemi vennero proposti, con piante rade in solchi mezzani, come praticasi fra noi, a solco molto profondo, che si riempie ali' epoca della prima rincalzatura, a fossette distanti un piede con una sola piauta, a fosselte distanti un netron quadirato, nelle quali al moilo Ame. ricano si coltiva un gruppo di quattro piante. Nell' anno scorso per invito della società Agraria di Bologna veunero sperimentati comparativamente $i$ quattro metodi suddetti che per la loro efficacia sulla produzioue dal seme, si possono disporre coll' ordine seguente $1 .^{\circ}$ Americano. $2 .^{\circ} \mathrm{A}$ fossetle. $3 .^{\circ} \mathrm{A}$ solchi profondi e mezzani: mentre quanto alla produzione dei fusti e foglie verrebbe per $10^{\circ}$ quello a solco mezzano, e si succederebbero: $20^{\circ}$ quelio a solco profondo: $3{ }^{\circ}$ quello a fossette: $4 .^{\circ}$ quello Americano, verificandosi dal primo al terzo la differenza del is \% in peso del seme, e dal $10^{\circ}$ al $40^{\circ}$ il r 2 per ceuto in peso dei fusti. Il sistema Americano dunque contribuisce ad aumentare la produzione del seme, che e il fine per cui si coltiva il Formentone; il sistema nostro sarebbe invece favorevole alla produzione dell' erba, del che è facile trovarne la cagione. Resta ora a determinare l' effetto dei sistemi suddetti sulle colture successive, ciò che verrà messo in evidenza dai futuri raccolti.

In questi ultimi anni, uei quali per la mancanza del vino 
gli spirití aumeutarouo siffattamente di preżżo, si léntò d' introdurre anche fra noi certe piante capaci di fornine mediante la loro distillazione. Fra queste la più importante per il clima nostro è il Sorgo zuccherino, giacchè la barbabietola per difetto d' umidità vi cresce poco, e si arrichisce meno di materie zuccherine Il Sorgo è una grigautesca graminacea che pochi anni fa venue introdotta dalla Cina, e per molti rispetti rassomiglia alla Saggina da scope; ma oltre le differenze organiche per cui $:$ botanici ue fauno un genere diverso ne è anche assai più robusta, elevando talvolta sino a 5 metri i suoi fusti che hanno il diametro di un decimetro alla base. In grazia appunto di questa robusta ve etazioue anche nel clima nostro riesce ad estrarie dal suolo l' umidità ed il nutrimento necessario, ed a dare prodotli considerevoli in terre comparativamente mediocri. La materia zuccheriva e contenuta nel fusto, le foglie prssono servire di uutrimento al bestiame, ed $\mathrm{i}$ semi che maturando aumeutano la secrezione dello zucchero, contro ciò che avviene velle altre piante che lo somministrano, possono essere utilmente impiegati nell' ingrasso dei majali. II Professote ed il suttoscritto sperimentarono l' anno passato questa coltura con sufflcieute successo, é ne ricavarono tno spirito privo di quell' odore disgustoso che accompagna sempre i prodolti tratti da altre sorgenti che il viuo.

Dal conto approssimativo che se n' è istituito, risulta che questa collura potrelibe fira noi riuscire emineutemente lucrosa.

Niuno ignora che duraute l'inverno i foraggi freschi eccitano gli animali a consunıare altri for ggi scadenti, di che si uutrono svogliataneute. Fra questi i migliori ei vengono somministrati dalla Barbabietola, giacchè le patate richiedono particolari diligeuze vella loro preparazioue, e le Rape per mancanza dell' umidità vecessaria ingrossano poco. Siccome però non è sperabile che il Contadino s' uuduca a coltivarle come si pratica altrove a spese della prediletta coltura del Formentone, il Professore volle sperimentare in quest' anuo se in grazia della mite temperatura dei nostri inverni si potrebbe coltivarle in raccolto eventuale dopo il frumento. L' esito corrispose perfettamente alla sua speranza e 1825 piante di Barbietole seminate in vivajo nell' Aprile, il $5 \mathrm{Lu}$ - 
glio veunero trapiantate sopra un mediocre lavoro dato alle stoppie. Soflersero l' aridità estiva senza perire, cominciarono ad iugrossare dopo le prime pioggie di Agosto sostenendo seuza detrimento nel suolo le forti gelate del Dicenibre e col peso medio di libbre 5 l' una, fornirono circa libbre 10000 di materia alimestare, equivalente per il sua valore nutritivo a circa libbre 2000 di Fieno.

\section{Ineoraggimenti mediante lo Studio di argomenti speciali}

La coltura arhorea è uno dei cardinì dell' Agricoltura uella uostra Ptovincia, dove le estati calde e sechissime, la poca profondità dei terreni, e la loro pendenza, rendono meno produltive le piante erbacee da foraggio e da seme. Ma questa foute privcipale della uostra ricehezza minaccia d' inaridire o per iguote ed indomabili malatlie, o per contrario ed insolito andamento delle stagioni. La malattia della Vite e dei Bachi da seta, la mortalità degli Olivi, richiamarono lin da principio l'attenzione dell' Accademia, la quale non solo procurò di raccogliere quei fatti che potessero illustrarue la -loria, ma altresì fu sempre sollecila a divulgare $\mathrm{i}$ rnezzi proposti per combattere od attenuarne le conseguenze.

\section{VIalattia della Vite}

Falli positivi, concordauti mostrarono evideutemente che l'Oidium Tukeri M., criltogama parassita dẹ fusti foglie e frutti della Vite, è la cagione precipua della malattia che distrugge da molti anui le uve, e fa perire gran numero di viti. L'Accademia nostra fu delle prime a riconoscerlo, cone risulta dalla relazione dei chiarissimi Marchese Petrucci e Profess Gralvani iuserita nel $\boldsymbol{x}^{\circ}$ semestre dell' Anno XII. ${ }^{9}$ delle Esercitazioni, e fiu dal 1852 in cui apparve la fatale parassita nel territorio della Pravincia, propose lo zolfo in pol. vere qual mezzo efficace per combatterne la diffusione. Ma 
non bastava di aver indicato il rimedio, conveniva acloperarsi per diffouderne la cognizione, mettere alla portata di tulti ordigni economici per spargerlo con unifornità sulle piante, conveniva insistere per vincere l' indolenza dei possideuti, e la proverbiale avversione dei campagnuoli a qualuucque iunovazione. L'Accadenia in fatti fece pubblicare dal benemerito Professore Galvani un' istruzione popolare per la Zolforazione delle Viti, diede i disegni degli apparati per eseguirla, chiese ed oltenue che la Casa Albani ponesse deposito di Zolfo in polvere a Pesara ed in Urbino. E tristo confessare che mentre nel Nord dell' Europa, nella Grecia, e fina vell' Agro Ro. nauo ricorrevasi all' efficace rimedio, d'oude il grande aumento nel valore dello Zolfo, e la grande esportazione che ue vien fatta per quei paesi, fra noi queste cure producessero pochissimo effetto, allegando i più di uon credere all' efficacia del rimedio, altri mettendo iunanzi la spesa grave della mano d'opera, il clima e la diversa coltura delle viti, altri iufiue anteponendo senza ragione plausibile alcuni rinedii suggeriti da un cieco empirismo o da considerazioni puramente leoriche. L' esperienze comparative fatte per ordine di Monsignor Ministro del Commercio sull' efficacia di questi riusedii e l' esame dei risultati ottenuti con lo Zolfo in diverse parti di Europa fornirouo all' attuale Professore dati necessarii per combattere qualunque dubbio sull' utile ed economico impiego di questo agente preservativo. Nella prossima primavera Egli pubblicherà una istruzione popolare intoruo alla pratıca ed alla utilità di zolforare le Viti, il che divenue ora tanto più necessario in quanto la malattia invece di audar progressivamente declinaudo, come erasi sperato, toruò ad infierire ngell' auno scorso con la primitiva violenza.

\section{IIIalattia dei IRachỉ dla Geta}

La coltura, dei Gelsi si è rapidamente estesa nella nostra Provincia, aumentando il valore dei terreni, e contribuendo indirettamente al miglioramento dell' agricoltura. Il prodolto che se ne ricavò negli ultimi anui fin veramente straordinario, e quautunque non convenga far asseguameulo sulla coutinua- 


\section{$-15-$}

zinue di una rendita si elevata, è jnuegabile che mercè di quella soltanto i nostri possidenti si trovarono in condizione di effettuare rapidamente migliorie che avreblero richiesto lunghissimo tenipo. Peró la moltiplicazione di questa pianta preziosa non distrusse ancora l' equilibrio delle colture, e fu ventura, giacchè la varietà di queste sarà ancora per lungo tempo fra noi la cagione principale della pubblica prosperità. Mentre estendevasi la coltura del Gelso riformavasi il sistema di allevamento dei Filugelli, imitandosi il meğlio di ciò che praticavasi in Lombardia ed evitandone $\mathrm{i}$ defetli, onde la riuscita degli allevamenti divenue meno incerta, $\mathrm{e}$ ad un tempo più economica, e le razze si migliorarono, al seguo che le sete nostre sui mercali di Torino, Mlilano e Lione, formavano una classe a parte ricercata per i lavori più delicati. Non è questo il luogo di esaminare se il sistema di allevamento che ue risultò potesse in progresso di tempo influire sulla salute del Baco, e se facendo vivere la larva in condizioni molte diverse dalle naturali, si andasse incontro al pericolo d'iudebolire la specie predisponendola a contrarre nuove malattie, od a soccombere sotto goli assalti delle antiche. Forse l' epidemia che da parecchi anni va disertando le Bacherie dell' Europa meridionale, in questa degenerazione della specie più che nell' influeuza delle stagioni trovò un mezzo ëfficace per diffondersi e perpetuarsi. Infatti la mortalità che più o meno si osserva attualmente in tutti gli allevamenti non è nuova, ma proviene da una di quelle antiche forme morbose che in modo accidcutale e sporadico osservaronsi quasi sempre in tulti gli allevamenti, ma che oggi ha preso tale esteusione da rendere inutili tutti i rimedi, oude parve migliore cousiglio combatterla rinnovando le razze con un seguito di allevamenti all'aria libera, ovvero traendo le ova direttamente da quei paesi nei quali il Baco nou fu sottoposto alla domesticità. L' Accademia che nell'ammo passato ebbe agio di seguire l'andamento delle molte razze asiatiche allevate nei possessi del Principe Albani prese già gli opportuni provvedimenti per avere in quest'anno il seme ehe due illustri Agronomi italiani recarousi a fabricare nella Cina, commise al Professore di allevare nella veutura prinavera qualcuna delle razze nostrali all' aria libera, non dislogliendo inai la sua attenzione da ciò che scrivevasi altrove su questo argomeuto che non solo iuteressa 
l'Agricoitura, ma che oggi è anche stretlamente collegalo con la prosperità delle città priucipali della Provincıa.

Ma se finora riuscirono vane le precauzioui ed $\mathrm{i}$ rimedi per combattere l'invasione di questa epidemia, la quale trasmeltendosi per via della generazione minaceia di distruggere la specie, un illustre naturalista Lombardo ha proposto un mezzo per distinguere uei Bachi da seta la semeuta buona da quella che fosse infetta dai germi della malattia predomiuante, e forse di qualunque altra. Dopo la bella scoperta di Agostiuo Bassi sulta causa del calcino, non era più possibile studiare le malattie del Baco nei soliti feuomeni esteriori, ma, segueudo I' esempio dato due secoli prima dall'illustre Malpighi, era divenulo necessario di ricorrere all' Auatomia, ed alla Chimica per riconoseere la sede dei diversi morbi e determinare le alterazioni che producono negli organi e nei principii inmediati oude quelli sono costituiti. Con tali aiuti la Patologia del Baco fece in pochi anui progressi importauti, e. si scopersero fatti singolari che un giorno potranno. coudurci a modificare sostanzialmente l'industria del setificio. Fra le molte cose che l' auatomia microscopica rivelò nelV'organismo del Baco, fu auche la struttura dei globuli del. sangue nello stato di sauità e nella malattia clie ora predomina. Iufatti il sangue del Baco sano è leggermente acido e formicola di corpicciuoli trasparenti del diametro di $1 / 20$ di milfimetro. Questi globetti hanno una specie di nocciolo che, come nel mucleo delle cellule regetali, sembra avere una figliuolanza di globettivi simili. Nei Bachi malati questi globuli del sangue spariscono o scemano, ed invece vi appariscono in gran numero corpicciuoli di forme diverse, che st muovono come sè fra loro, ora si altirassero, ora si respingessero, siugolare e curioso feuomeno che salta subito all' occhio delle persone anche del tulto iguare dell' uso del microscopio.

Il Dottor Carlo Vittadini di Milano nel marzo r859 propose di applicare l'osservazione di questo fenomeno conosciuto a distinguere il seme dei Bachi infetto dal sano. Posto in fatti che la presenza di questi corpiccìuoli indichi uno stato anormale nell' organismo del Baco, qual criterio più sicuro. puó aversi per giudicare della bontà di una semente, e prevederue l' esito che farne uascere una piccola quantilà sui prinzi 
di marzo, osservando por se nel sangue delle piccole larve s' incoutra il sintomo fatale? E qual altra conclusione potrebbe trarsi dall' esistenza di quello se non che , Bachi sono malati, e perciò che il seme da cui provengono non è buono?' Affinchè però la comparsa di questi corpiciuoli non si dovesse attribuire ad una alterazione proclolla uella salute dei Bachi dalla nascita anticipata il Vitladini propose di osservare direttamente il seme, giacchè se è infetto i corpicciuoli oscillanti comprono immancabilmente uell' embrione, e uelle materie destiuate a nutrirlo appeua comincia il suo moto di evoluzione, o, come volgarmente si dice, la semente connincia a risentirsi. Le pazienti osservazioni istituite dal Professore, e dal sottoscritto per verificare l' utilità del metodo di osservazione proposto non lasciò nell' animo loro alcun dubbio sulI' effeacia e sicurezza del medesimo, per cui non dubitano asserire che sementi, le quali fossero prive di siffatli corpuscoli potrauno beusi fare pessima prova o per difello di cure durante l'allevameuto, o per altre cagioni ignote; ma essere difficilissimo per non dire impossibile che si otlenghino buoni risultati dalle sementi in cui quelli si osservarouo, giacchè $i$ Bachi per poco che ue siano infetti dalla nascita, nou potranuo protrarre la loro esisteuza sino alla formazione del bozzolo. Questu mezzo però offre in pratica, qualche difficoltà, possedendosi da pochi un buon microscopio, che poi tutti nou saprebbero adoperare. Affinchè però la nostra Provincia possa fin da quest' anno cavar partilo dell' importante scoperta, verrà dal Professore vel prossino Marzo pubblicata us' istruzione intorno al modo di farne uso; mentre poi egli si presterà gratuitamente ad esamiuare quelle sementi che gli venissero prescutate uella scuola duraute tutto il mese di $\Lambda$ prile.

\section{Ploxtalità đlegli Olivi}

L' Olivo formò in passato la riceliezza delle nostre colbine calcari, massime in vicinanza del mare che sembra favoreggiarlo. Da parecchi anni però per effetlo di freddi rigorosi e precoci, e in grazia della prediletta coltura del Formentone introdolta uell' Oliveto i prodotti diminuirouo, mentre si ac: 
crebbe la mortalità delle piante. Si credette allora di portarvi rimedio, esagerando la potatura senza riguardo a mantenere un convenieute equilibrio tra le froude e le radici, il che fu cagione di nuavi danni. L' accademico signor Tereuzio Nardi inmaginò di combattere tale errore addestrando compagnie di potatori nei propri Oliveti, e cedendoli poscia ai possidenti limitrofi suoi amici. $\mathbf{L}$ ' esito corrispose alla sua aspettativa, e quantunque il tentativo venisse praticato in proporzioni ristrelte non fin senza effetto per alcune parti del terrilorio della Provincia che trovano nell' Olivo la sorgente principale della lara ricchezza.

\section{Emsetif nocevoli agli Alberi da Frutto.}

Anche le nostre piante da frutto, massime i Peri ed i: Meli negli anni precedenti furono devastati da alcuni Bruchi, che rodendone tutte le foglie nel mezzo dell' estate non sola eagionavano la perdita dei raccolti, ma potevano ancora in progresso di tempo far perire le piante. L'Accademia fu soliecita di pulblicare nel fascicolo I. ${ }^{\circ}$ dell' anno X11. ${ }^{\circ}$ un' istruziove del chiarissimo Professor Gralvani per distruggere questi insetti distribuendola gratuitamente ai Possidenti e Fatlori che ue fecero richiesta.

\section{Allevamento delle Ap:}

II deplorabile sistema di diboscamento, e l'estensione. data alle colture granilere è stata cagione che l'allevamenio. delle Api andasse progressivamente scemando nel nostro terrilorio, come lo prova la diminuita esportazione del mele. A rimettere nuovaniente in onore questa industria rurale l' itlustre uostro Collega Marchese Petrucci nel più volte citato fascicolo delle Esercitazioui pubblicò una memoria intoroo al modo di cavar il mele senza uccidere le A pi, pratica barbara che deteriora la qualita del prodolto e forma uu osta- 
colo grautissimo all' esteusione di questa industria, nueutre il nostro Collega Dott. Achille Carnevali pose un Alveare considerevole vei suoi predj di Mondaino, goveruaudolo secondn i buoni principii dell' arte.

\section{Governo delle Acume.}

La coudotta delfe acque è strellanente collegata allo sviluppo dell' Agricoltura e del Commercio non meno che alla pubblica igieue. Il regime delle acque nella nostra Provincia è vizioso, e potrebbe essere perfetto per l'elevazione delle pianure sul livello del mare, e la ripartizione delle aeque prodotta dai monti e culli numernsi che la intersceano da ogni lato. Infatti per mancauza di stabile alveo lungo le sponde dei torreuti vanuo perduti grandissimi spazii, ove appunto il terreno sarebbe più fertile. Tale perdita è particolare ad alcuni ternitorj soltanto, ma vi è un allro errore, i cui effetti si estendono su tutta la Provincia, impoverendo i territorii di collina, e preparando ostacoli grandissimi all'igiene pubblica dei paesi di pianura ed alla libera navigazioue dei porti. Infalti la trista pratica di portare li scoli dei campi ove si trova il maggiore declivio, toglie a poco a poco ai terreni quella regolarita di superficie, senza di cui i lavori riescono più costosi e meno efficaci, cagiona la fiequenza delle frane che poi si convertono in burroni, reudendo injpossibile qualunque coltura sopra vaste superficie, favorisce le depredazioni delle acque, che spogliano a poco a poco le colline di tulta la terra vegelale la quale poi depositandosi nell' alveo dei fiumi presso le foci impedisce lo scolo delle pianure superiori, con danno della pubblica salute e della navigazioue. L' Accademia Agraria nel 185 I studiò diligentemente i mezzi di suttoporre il goveruo delle acque ed $\mathrm{i}$ dissodamenti ed un ordinamento che senza ledere il libero esercizio del diritto di proprietà lo accordasse meglio coll' iuteresse generale presente $€$ la sicurezza dell' avvenire. Frutto di questi studi fu un progetto che, ottenuta la sanzione delle Congregazioni Governative, dei Tribunali, e di quasi tutti i Municipi della Provincia venne nel 1853 presentato a Monsignor Ministro del Com- 
mercio e Lavori Pubblici, che si limitò ad accoglierlo e far. sperare che in breve verrebbe pubblicata una Legge in proposito applicahile a tutto to Stato. La morte del Ministro sopravvenuta pochi mesi dopo forse fu cagione che la speranza. non si avverasse, per cui in quest' anno venne ripreso lo studio dell' importante questione da alcuni membri dell' Accademia coll' iutendimeuto di ricorrere all' interesse privato per la formazione di speciali consorzi nei diversi territorii, chiedendo solo al Governo l' approvazione dei medesimi. Il Professore in sieme al siguor Domenico Guerrini compilarono ancora uno schema di progetto all' effettuazione del quale è desiderabile che si possa metter mano al più presto.

\section{Incoraggiamento mediante i premi.}

Due premiazioni del bestiame hanno avulo luogo nella nostra Provincia, dopo che il Consiglio raccomandò alla Accademia di ricorrere a questo mezzo onde promuovere il niglioramento delle nostre razze. Nei Programni di concorso che si allegano alla Lettera D. l'Accademia lasciò aperta la via a tutti i Possidenti della Provincia, assegnando premi per le razze di monte e piano, e per il bestiame minuto che meglio rispondesse alla condzzione del territorio che l' iuviava. Tali concorsi però misern in evideuza quanto poco interesse generalmente si porti lia noi alle cose agrarie; e tanto a $\mathrm{Pe}$ saro che a Urbino, dove il Municipio fece ogui potere per crescere decoro alla Festa, gli esponenti furono pochi, e tranne qualche onorevole eccezione, non venue presentato ciò che pur si trovava di meglio nella Provincia. L'Accademia continuerà nella misura dei mezzi di cui dispoue a pronuovere con premi il miglioramento dei nostri bestiansi, conlidandosi nell' azione del iempo e nell' efficace cooperazione delle SS. VV. Illustrissime, affuchè l'opera sua trovi nei diversi territori migliore corrispondenza, senza di cui non è possibile risvegliare l'emulazione fra i diversi possidenti, il che è il fiue principale di cosifatte solennità.

Eeco, o Signori, ciò che l'Accademia è andata operando per couformarsi allo spirito dei suoi Regolamenti e secondare 
le provvide intenzioui del Consigho Provinciale che la protegge. Dell' effelto de' suoi lavori potrà solo essere giudice il tempo, giacchè l'Agricoltura di un paese non si cambia di un tratto, ed il sistema che essa adolta dopo lunghe incertezze. e l'incessante aiuto dell' intelligeizza e dei capitali anzichè essere la conseguenza di preconcette opiuioni, è piuttosto il risultato della condizione economica di esso, dell' istruzione dei suoi abitanti, delle abitudiui, e dell'indole loro. Iufatti le innovazioni si fanno cou lentezza, si succedono, ma uon si combiuano in modo da costituire sio da principio un sistema completo d'Agricoltura bello e formato, ma una innovazione ne fa nascere un' altra, e la necessità di coordinarle, conduce in fine ad un sistema d'agricoltura affatto nuovo del quale solo ai posteri è dato di far ragione. Se le SS. VV. Illme vorranno portare la loro attenzione sui mutamenti che già si sono prodotti, sia mediante la maggior estensione data alla coltura dei foraggi, sia nella pratica dei lavori, sia nell' allevameuto dei bestiami, sia uell' industria della sela, vedranno che la Provincia nostra camina in questa via di progresso, aperta dall' Accademia cou i suoi lavori, con l' esempio dato da' suoi Membri, e con gl'incoraggiamenti ond' essa fu sempre larga a quanti riconobliero che l'A gricoltura è l'unico foudamento della uostra prosperità.

Tali cose l'Istituto nostro pote in gran parte operare mediante la protezione della Provincia, la coutinuazione della quale porto sperauza che non sarà per venirgli meno nell'arveuire.

$\mathrm{E}$ in tale fiducia ho l'onore di protestarmi con profondo ossequio.

Delle SS. VV. Illine ed Eccme

Pesaro I Febbrajo I 860 :

Agli Illmi ed Eccmi Signori

I Sigg. Consiglieri della Provincia di Pesaro e Urbino

Devmo ossequmo Servitore

iL PRESIDENTE DELL' ACCADEMIA

GIACONO MATTEI

II ff. di Seg. - LUrGI GUrd 
Lettera del Professore di Agricoltura al Presidente dell'Accademia intorno all' andamento della Scuiola.

\section{Illustrissimo Signore}

F

ssendo questa la prima volta in cui dopo molti mesi si riuuisce la nostra Accademia, ho creduto necessario di rasseguare alla $\mathrm{S}$. V. Illma una breve relazione della Scuola di Agricoltura che gli Onorevoli miei Colleghi vollero aftidarni con una fiducia che mi ouorava altameute, ma che al tempo stesso m' impose il dovere di porre tutto lo zelo e la lealtà di cui sono capace per supplire al difetto dell' ingegno e della dottrina, che in me sono troppo inferiori all' assunto. Io prego pertauto la S. V. Illma di sottoporre ai medesimi queste poche pagine nelle quali ho esposto il concetto che mi sono formato del fine e dei limiti dell' insegnamento Agrario, ed ho messo insieme l' orditura generale del Corso che sono andato intessendo in questi dieciotto mesi, affinchè giudichino liberamente se il melodo da me addoltato possa realmente servire a generalizzare i principii di questa scienza, e ad indurre negli animi degli Uditori quella persuasione mediante la quale soltanto i precetti dalle discussioni Accademiche possouo trapassare nell' esercizio e nella pratica viva dell' Arte.

L'Agricoltura fu già paragonata da alcuno ad un Area vastissima, il cui perimetro fosse limitato da quasi tutti i rami dello Scibile. Tale paragone non sembrerà esagerato a chi ponga mente ai reciproci legami di questa scienza con le altre umane discipline, ed alle numerose attinenze che la collegauo con le diverse parti dell' edifizio sociale. Infatti l'Agricoltura si propone di ricercare i modi più acconci per ricavare dalla terra molti e buoni prodotti con poca spesa, e di spacciarli con profitto al fine di rendere la vita umana più 


\section{$-23-$}

vomoda e più grata. Essa perciò deve indagaie quali sieno lc piante utili all' uomo, ed i modi meglio arldatlati, e piì écononici di coltivarle; deve ricereare quali sieno, ed in qual maniera si debbano allevare gli animali che cou la loro forza ne alleviano le fatiche, che con i loro prodolti concorrono a nudrirlo, e che somministrano alcuue materie prime per le sue industrie; essa finaluente deve determinare yuando c: come convenga trasformare certi prodotti grezzi in prodolti mauifatturati di piû facile conservazione e trasporto, o di vendita più lucrosa. Perciò meutre uelle altre industrie l'uomo si trova alle prese con un oggetto soltanto, l'agricoftore invece ha da fare con una varietå considerevole di materie, tener dietro ai numerosi rapporti delle medesime, regolando la distrihuzione degli esseri viventi su quella superficie di terra su cui estende il proprio dominio; modificando, entro certi límiti, l' orgauica conformazione di essi secondo i proprii bisogui, e in corrispoudenza alla composizione del suolo, allo stato dell' aria, all' abbondauza delle acque, alla natura delle piante, e degli animali, non meno che alla ricchezza, alla popolazione, ed alla generale prosperità di un paese. Nè ciò basta, che le diverse parti di questa vasta istrapresa debbono essere in tale stretto rapporto, e per tal modo proporzionate fra loio, che nessuna ecceda le altre in modo da turbare l'armonia dell\}, insieme, che niuna si possa dire inutile e superflua, ma tutte concorrino all' unico fine di aumentare la rendita netta del suolo, o coll' accrescere la quantità dei prodotti, o col diminuire la spesa per ottenerti. Ora tutto ciò non può farsi con certezza di buon successo senza possedere idee precise intorno al modo col quale gli esseri viventí si nutrono, e si riproducono, e senza conoscere a pieno la proprietà delle terre e dei climi. Che si direbbe di un Ragioniere il quale perchè ha appreso le regole di tenere un libro di amministrazione in partita doppia, si reputasse in grado d'intraprendere il commercio di materie che non conosce, o di uu Muratore, che avendo inalzato una colonna ben a piombo si argomentasse di costruire un ponte senza alcuna cognizione di disegno, di geometria, e di mecanica? Costoro non potrebbero operare se won a caso, senza regole, e senza priucipii, e incapaci di rendersi conto della propria gestione, non saprebbero valutare le difficoltà, uè trovare i mezzi di superarle, per modo che la 
riuscita della loro intrapresa, dovrebbe aversi in conto di miracolo anzichè attribuire alla saggezza delle loro previsioni. Eppure l'agricoltore che uon ha una cognizione precisa del suolo, delle piante, e degli animali, che non sa valutare i rapporti della sua industria con le condizioni del paese e del tempo in cui vive, non procede in modo diverso da quelli, e sia che corra avidanieute dietro a tutte le novità, sia che rimanga servo di una cieca consuetudive, opererà sempre a caso, e couverrà che rimanga contento dei risultati che il caso può darè.

Ma se niuno oggi ardirebbe porre in dubbio l'utilità dell' istrużione agraria, variano però moltissimo le opinioni intorno al modo di compartirla. Infatti alcuni vorebbero che l' iusegnamento frsse unicamente rivolto ai contadiui, apoggiandosi del tutlo su quella pratica che ha la sua dottriva, ma non è scienza per questo, giacchè se può dare utili suggerimenti per la specialita dei casi, non è atta ad abbracciare le moltuplici relazioni di un azienda rurale, e di risolvere con felice esito la naggior parte delle difficoltà che occorrono nell'esercizio dell' arte. Costoro somo persuasi che con lo studio e le meditazioni dei dotti, l' agricoltura non si possa correggerè nê migliorare, ed incapaci di affrontare le difficoltà uumerose che tengono dietro alle più semplici modificazioni delle pratiche agrarie, sostengono la superiorità di tuta i $\mathrm{i}$ metodi antichi, perseguitando con i loro dileggi le più 'utili inuovazioni. Nè questi soùo i soli che combattano l' influenza delle scienze e dei loro progressi nell' inseguamento dell' agricoltura. La scienza agraria è nata e si è svolta primieramente sotto lo stimolo del bisoguo e del genio operoso delia stirpe A uglo-Sassone nelle regioni settentrionali di Europa. Non máncano perciò uomini d' ingegno, i quali, riconoscendo il grande incremento clie in quei paesi ebhe l'Agricoltura poichè venne fecondata dalla scienza e dai capitali, per la diversită di condizioni fra il mezzogiorno ed il seltentrione, ritengono impossibile e dannosa ogni mutazione delle nostre pratiche antiche. Altri al contrario vorebbero che l' insegnamento non uscisse dai principii assoluti della scienza, senza curarsi disuggerire le norme per giudicare dove e come possano l'innovazioni utilmente adattarsi, e dimenticando che sebbene la scienza, sia un faro a cui debbano sempre essere rivolti gli 
ocehi dell' agricollore, per tradurre $i$ principii nella pratica urcorra tale un accorgimento prudente e una sapiente riserva, tale un colpo d'occhio sicuro, od una tenacità di proposito le quali dilicilmente s'incontrano in coloro che con $\mathrm{i}$ confronti dell' esperienza uon poterono conoscere come i suggrerimenti della scienza passando dall' astratto al concreto, $110 n$ riescano sempre egualmente utili come sono egualmente veri.

L' amore di sistema trae in errore i fautori di ambedue queste opinioni estreme. In falti la scienza nou è talmente sicura di tutte le sue teorie da potersi ritenere maestra e direttrice infallibile dagli agrarii interessi, e la pratica senza la scienza diviene testarda e dispregiatrice di ogni progresso che nou trovi un riscontro nelle pratiche autiche. E vecessario pertanto che la scienza e l'arte si diano reciprocameute la mano onde ricavare abbondauza di prodotti dal suolo con poca spesa. In questa unione feconda la scienza mette a servizio dell' arte ciò che l'umano intelletto ha saputo acquistare di più positivo penetrando nelle proprielà della materia, e nei segreti della vita, mentre la pratica, subordinata alla scienza ma nou fauatica della sua scorta, modifica a seconda del clima, delle condizioni economiche del paese, e dei capitali del coltivalore $i$ principj assoluti ed inflessibili di quella. Tale insegnamento però siuchè il beueficio dell' istruzione uon sarà penetrato nelle canjpagne non potrà essera rivolto al contatadino, che che per la nativa ignoranza non sarebbe disposto a far buon viso ai dettami della scienza, e che col nostro sistema di mezzadria sarebbe privo dell' autorità e dei mezzi di operare da se stesso le opportune inuovazioni. La nostra agricoltura, rimasta finora allo stato di semplice mestiere, in grazia del fino discernimento del nostro popolo, raggiunse nell' esattezza e diligenza delle faccende campestri tutta quella perfezione di che può essere capace un' iudustria abbandonata a sè stessa uel seuso assoluto della parola. Pcrò se in tal modo ne venue quell' ameno giardino delle uostre campagne, le quali spesso sono tanto più ridenti per arte quanto sarebbero per natura piì sterili e desolate (1), è fatto innegabile che questo sistema di agricoltura si appoggia tutto sulla fertilizzazione atmosferica, e che il sussidio dei concimi uon figu-

(1) Ridolf Lezioni orali d'A Agric. Lez. I. 
ra în generale che in debolissima proporzione. Consegueuzà di esso è una produzione mediocre accompagnata da un progressivo impoverimento del suolo; onde avviene che una parte dei capitali impiegati per mettere in frutto la terra, rimanghino seuza adequato compenso, e si considerino come scompar'si. II coloni", che è cointeressato soltanto precariamente uella rendita del suolo, soddisfatto della propria mediocrità, non cura, e nou ha un interesse proprio per rimediare a questo danno i cui effelti vánno progressivamente aumeutando, e potranuo provvedervi solo i possidenti, i quali come proprietarii del térreno hanno interesse diretto di ricercare i mezzi per ricavare una rendita delle somme anticipate alla terra, e per procurare che nel più breve periodo di tempo veughino ammortizzate. Oltre che i possideuti terrieri avendo nella coltura dell' ingegno un ajuto potente per scuotere il giogo degli in. veterati pregiudizj, trovano anche cowe proprietarj del suolo i capitali occorrenti per effettuare le miglioriè necessarie, mentre da ultimo mediante la loro autorità, mantenuta dalla natura del coutratto di mezzadria e dalla tradizione, riescono a fare adottare dai contadini le pratiche nuove di cui diffidauo, come il cieco diflida di ogni nuovo sentiero. Infatti la storia di tutte le nazioni $c^{\prime}$ insegua che la riforma e i miglioramenti nell'arte coltivatrice, non si dovettero mai al contadino di mestie. $\mathrm{re}$, ma a persoue istruite, e beve educate che operarono con riflessione e secondo un sistema naturalmente ordinato, perochè la destrezzà indispensabile, i mezzi occorrenti, e le cognizioni estese e precise che fa meslieri di possedere per ordinare le diverse parti di un' azienda rurale; non sono alla portata dei pratici coltivatori, che operano cone manuali, lasciandosi guidare ciecamente dalle ricevute costumanze.

L'Agricoltura pertanto mediante l'influeuza delle scieuze che prese per guida è uscita oggi dalla categoria di semplice mestiere, prendendo posto fra quelle nobili discipline che meditano sulle leggi della natura per trarue profitto. Al pari della Medicina e della Chirurgia essa applica alla ricerca e al governo di certi fenomeni naturali i metodi d'investigazione, ed i priucipj di parecchie scienze pure, per cui è necessario premettere la coguizione sommaria di questi principj allo studio delle materie che ne costituiscono l' oggetto speciale. Avviene perciò che l' insegnamento dell' agricoltura nelle 


\section{$-27-$}

cillà di provincia riesca ben altrimenti difficile che nelle Università o nei paesi, nei quali l' istruzione secondaría essendo meglio compartita, non vengono ammessi a questa scuola se mon i giovani che hanno compiuto il corso elementare delle Matematiche e delle Scienze uaturali. In questo caso posseg. gono i principj foudamentali dell' arte agraria, per cui sono in grado d'inteudere il linguaggio preciso della scienza, conoseono i legami che connettono i tre regni della natura nell' iuteresse rurale, e ciò che più importa, essendo assuefatti a ricercare lé cagioni occulte dei fatti ed a coordiuarli e a confroutarli per dedurne i rapporti, posseggono to strumeuto opportuno, il vero metodo per esercitare con buon successo la professione di agricoltore, e assicurarsi della enorme diversità che passa tra la produzione ottenuta, colle pratiche razionali, che la scienza consiglia, e quella che offre attualmente la maggior parte dei nostri terreni. Ma nelle piccole città mancauo le scuole per tali studj, essendo già un grau fatto se i giovani poterono compiere un corso elementare di Fisica, e di Matemalica ; per eui conviene che il maestro si restringa a dar ricette che riescono o falliscano secondo le persone ed $\mathrm{i}$ luoghi in cui si pongono in pratica, ovvero si tolga l'improba fatica d'inseguare i principii di molte di tali scienze uelle loro attinenze coll' arte agraria, facendo però a ciascuna di essa una parte tale, che nou alteri l' ecouomia generale del Corso, il quale uel mio caso particolare è limitato a tre auui.

Le piante vivono nell' aria e nella terra, lo studio di questi due ambieuti, come li chiama l' illustre Prof. Cuppari, è per solito il punto di partenza di tutti i corsi d' agricoltura. Ma come parlare dei rapporti dell' atmosfera col suolo, e delle proprietà fisiche e chimiche vel terreno agrario a chi ignora del tutto la qualità e l'indole degli elementi da cui l' aria e la terra souo costituite?' Mi parve perciò che vell' ordine logico delle idee i principii di una Chimica inorganica ristretta ai corpi minerali che hanuo una relazione immediata cou l'agricoltura, fossero come la porta per la quale convenisse mettere $\mathrm{i}$ giovani nello studio di questa, a meno che ad una istruzione soda e capace di buoui frutti non si preferisse quella vana scienza di parole atta a fare dei presuntuosi, che in ogai cosa riescono sempre peggio degli ignoranti.

Conosciuta la qualità e le leggi con cui si associano i 
corpi elementari che costituiscono l' aria e le diverse materié; onde risulta il terreuo agrario, fu agevole insegnare come si eseguiscano le diverse operazioni analitiche, che rivelando la composizioue chimica e le proprietà fisiche dei diversi terreni, forniscono dati sì preziosi iutorno al valore dei medesimi ed al nodo di coltivarli. Però l'agricoltura non è seienza speculativa che debba restrivgersi al solo esame dei fatti, ma come scienza d'applicázione deve rivolgessi principalmente alla ricerca dei mezzi per dominarli, modifieando, in ordine ai prodotti che vuol conseguire, le propietà, e la natura dél suolo nel modo piic efficace od economico, nel che sta appunto la perfezione dell' arte. Ora le condizioni di un terreno si modificano mediante la condotta delle acque, mediante i lavori aratori, mediante l'irrigazione, i correttivi terrosi, l' avvicendamento delle colture, $i$ coucimi $\dot{E}$ perciò che in questa parte del corso trovarono il loro posto naturale, $i$ principii generali, $10^{\circ}$ del governö delle acque in piano ed in colle con l' indicazione delle leggri civili che ne modificano l'applicazione; $2{ }^{\circ}$ Le norme generali per le colmate di piano e di monte, e per la fognatura ; 3 ' $^{ð}$ La descrizione degli strumenti agricoli, vanga, zappa, aratro, erpice, estirpature ecc. con l'arialisi del loro modo di agire e gli esercizii per apprendere a maneggiarli; $4^{\circ}$ Le regale per $1^{\prime}$ uso degli emendamenti, e pei la debbiatura ; $50^{\circ}$ Fiualmente il tratt to dell' irrigazione con le norme per la formazione delle marcite. Affinchè poi i giovani fossero in grado di giudicare-dove e come queste inuovazioni potessero utilmente adottarsi aggiunsi intorno ciascuno dei predetti argomenti $\mathrm{i}$ titoli di entrata e spesa calcolati sul valore reale della mano d'opera e sul frutto corrente del denaro uel nostro terrilorio.

L' applicazione di tali regole, l' uso di tali ageuti modificatori del sublo è però subordinato alla natura delle piaute che si vogliono coltivare. Oltrechè mal si potrebbe far ragione dell' efficacia dei diversi concimi, e di certe successioni di coltura prima di avere appreso quali sieno i principii elensentari, ed immediati delle piante, quale sia la siruttura dei loro organi, e quali le fuuzioni che questi esercitano nell' economia della natura. Pertanto cominciai nel corrente auno que. sta seconda parte del Corso con alcuue lezioni di Chimica Vegetale, nelle quali però misi da parte ogni lusso di fornole 
e di teorie e mi restriusi soltanto $10^{\circ}$ all' esame delle materie minerali che si riuvengono nelle ceneri delle piante domestiche più importanti, onde ricavarne utili norme per la suecessione delle culture nell' avvicendamento; $\mathbf{3}^{\circ}$ allo studio dell' indole, e delle successive trasformazioni di quei principii immediati delle piante, che alimeutann le arti agricole; $3 .^{\circ}$ alle norme generali per queste arti medesime, cioè per la fabbricazione del vino, del carbone, per l' estrazione dell "nlio per la preparazione delle fibre tessili del lino e della canapa; $40^{\circ}$ alle regole per la pratica del sovèscio; $50^{\circ}$ alla teorica della formazione dell' humis.

Terminato lo studio dei principii onde risultano le pianti passai ad esporre l' essere loro allo stato vivente. Però le mie lezioni di Botanica si restrinsera a quel tanto che l'arte agraria ha realmente bisogno di conoscere per i suoi fini, per cui con tale intendimento, compiuto lo studio degli organi elementari, non presi ad esame nell' Organografia propriamente detla che gli organi principali, allargaudomi invece nel discorso delle loro funzioni, onde dalla completa cognizione di queste, ni fosse agevole ricavare norme razionali per la potatura degli alberi fiuttiferi, per il governo dei hoschi, e per la moltiplicazione artuficiale delle piante mediante la talea, il margotto e l' inuesto. Cousiderando più l' indole svariata del nostro territorio, e le differeuze notabili di clima fra le pianure del littorale Adriatico, e le coste dell' $A_{\text {ppenino e della }}$ Carpegua mi parve non inutile fatica di consecrare due lezioui ad esporre i limiti geografici della naggaior parte delle nostre piante donestiche da leguo, da foragrgio e da seme.

is già terminata anche questa parte del Corso, la quale. conliene in potenza, come direbbero i mateunatici, quasi tutte le leggi dell' Agricoltura Speciale. Siccone però. questa non può esercitarsi senza concini e lavori, le quali due conntizioni presupongono l'esistenza delle macchine che debbono produrli, cioè degli Animali, mi propougo di fare nelle poshe settinane che restano prima delle vacauze alcuue lezioni di Chimica Animals, toceando altresi brevemente degli organi. e delle funzioni del movimento e della autrizione nelle sperie allevate per i hisogui, e le speeculazioui dell' arte agraria. La qual cosa mi pare urcessaria tanto per gittare le fondamenta delle pratiche di allerausento e d ingrasso che dovrauno 


\section{- 30 -}

esporsi nella Pastorizia, quanto per dedurne uвa teorica sema plice ad un tempo, e completa intorno al valore comparativo dei diversi concimi, e al modo di prepararli, e consegnarli al terreno.

Possedendo il criterio per giudicare delle qualità di us terreno e conoscendo i mezzi di modificarlo, avendo idee chiare sulla costituzione delle piante, e l' iudole dei loro prodotti, e sapendo da ultimo quel tanto che basta intoruo all'orgauizzazione degli animali domestici, parmi che il giovane possegga quelle verità fondamentali che dovranno essergli di scorta nella pratica dell' arte, alla quale resta che venga aviato coi traltali dell' Agricoltura Speciale e della Pastorizia, che costituirauno la terza ed ultima parte del Corso.

In questa, passate in rassegua tutte le piante coltivale, ed i titoli d' entrata e spesa per ciascheduna di esse nei territorii principali della Proviucia, veduto il modo di ordinare praterie temporanee e permaneuti, fissate le norme generali per l' allevamento ed ingrasso del bestiame, e iudicati i caratteri esteriori che rilevano un' attitudine piuttostochè un' altra, si coronerà l' edificio pazientemente innalzato con la teorica degli avvicendamenti, proponendo quelli che secondo le condizioni fisiche ed economiche delle parti principali della Pro. vincia, potrebbero utilmente essere adottati. Il corso si conchiuderà con le regole geuerali della contabilità Agricola, la quale, secoudo il mio concetto, non solo deve esscre il mezzo per giudicare colla misura del tornaconto l' esito delle singole operazioni, ma dovià anche offerire una maniera di prospello siutetico di tutta l' azienda, per modo che i diversi fattori della produzione essendo distribuiti in grandi classi apparisca evidentemeute il legame da cui sono collegati e si possa acquistare facilmente un idea chiara di tulto l' insieme. La qual cosa mi sembra tauto più necessaria, in quanto per la buona riuscila di un intrapresa Agraria non basta che ciascuna delle diverse operazinni veuga eseguita secondo le regole dell' arte, ma è necessario che queste siano collegate fra loro in modo che mellendole a riscontro non ve ne sia alcuna che turbi l' armonia dell' insieme, e dall' esame di una sola di esse si possa giudicare di tutle le altre. Mediante questa maniera di sintesi si evitano facilmente quasi tutti i pericoli e le difficoltả che s' incoutrano da chi voglia introdurre qualche innovazio. 
ne in agricoltura, giacchè potendo queste essere considerate prima isolatamente e poscia in ordine all' insieme di cui debbono far parte, il calcolo dell' intrapresa riesce chiarissino, e si possano prevedere tutte le mutazioni e le spese che saranno la conseguenza di quella prima innovazione. Perciò tulti gli Agronomi si accordano nel riguardare un buou sistema di coutabilità come uno dei fondamenti principali per il buon governo dell' azienda rurale, attribueado in gran parte alla imperfezione delle nostre scritture, se l'arte agraria fra uoi uou progredi ancora come aṿrebbe potuto.

La S. V. avrà rilevato clse questo Corsa di agricoltura, di cui ho tirato per così dire $\mathrm{i}$ primi stami, è strettameute siutetico. Procurai di seguire la stesso metorto vella esposizione delle diverse parti di esso perchè la sintesi mi sembra più acconcia quando si tratti d'. inseguare una scienza preudeudo le relative cognizioni al punto in cui sona, e non s'intenda d'istituire nuove ricerche, nel qual caso nou si può abbandonare il metodo analitico.

Cosi per esempio uella Chimica Vegetale sono partito dall' esame dei principi inmediati del seme per arrivare, come ha fatto Malaguti, allo studio dei principi che costituiscono la piauta adulta, o che sono un prodolto delle sue funzioni. Solo uella Botanica ritenni più utile procedere aualiticamente mediante lo studio di alcune delle piạte domestiche più iunportanti, e procurando sempre di rendere evidente la dimostrazione, o con l' esame della pianta vivente, o cou l'aualisi microseopica, o con $i$ disegni a tale effetto da me stesso rilevati. E poiche il discorso è caduto sulla Botanica reputo necessario accenuare, che a bello studio ho ommesso del tutto la Glossnlogia, la Tassonomia, la Fitografia, come vella Zoologia Agraria nou parlerò punoto dei diversi sistemi di olassilicazione, i quali sono l'espressione dello stato delle scienze. in una data epoca. Avendo sempre aborrito da quella istruzione superficiale, che sopracarica la memoria di parole senza arrichire la meute di un' idea, mi parve meglio di ommeltere queste parti del tulto che parlarne incompletamentc. Da un altio canto che imporla all' Agricoltore di appiendere le regole per descrivere esaltamente una nuova pianta, quale utilità può ricavare dal conoscere la storia dei diversi sistemi 
di elassificazione e dall' entrare uelle spinose questioni della determinazione, e stabilità delle specie?

Nel disegno che sono andato abbozzando, i miei Colleghi avranno rinarcato che l'esposizioue delle dottrine viene spesso interrotta dalle regole per esercitare cerle arti che ue dipeudouo, come per esempio la storia della fermentazione viene interrotta dalle regole per la fabbricazione del pane, e del vino, e lo studio dei corpi grassi dai principii generali dell' oleificio ; che uella Botanica all' esposizione delle funzioni fisio'ogiche, seguoun le norme per la condotta degli alberi fiuttiferi, come uella Chimica Auimale allo studio delle secrezioni seguirà la pratica del Caseificio, per cui a prima giuuta sembra mancare quell' ordiue severo, e quell' armonia di parti, che è il fondamento di qualunque scieuza. Ma io mi sono condotto ad ahbracciare un tale sistema per due ragioni: $10^{0}$ perchè nella esposizione dei principj delle scienze pure su cui riposa l'arte agraria le menti non perdessero mai di vista il fine pratico di questi studii ch' è l' agricoltura. 2. ${ }^{\circ}$ perchè i giovaui fossero tratti a tocear con mano come la professione di agricoltore e le arti che ne dipendono non solo si deducano da principii scientifici, ma nou sia dato di esercitarle utilmente se non adoperando $\mathrm{i}$ metodi stessi che le scienze pure impiegano nelle loro ricerche, vale a dire l' osservazione ed il calcolo, come insegnarono tulti i grandi agrononi da Thaer a Gasparini e Ridolfi.

Per comodo degli scolari raccolgo iu brevi note i principii esposti con maggiore ampiezza nella lezione orale, procuraudo di nou perdere mai di vista le condizioni speciali della nostra Proviueia, sia dal lato elinatologieo, sia dal lato tellurico, sia finalmente dal lato economico, in modo che ne risulti un compendio, nel quale l'arte diretta dai principii generali della scienza nou divenisse fanatica della sua scorta dimenticando l'osservazione delle pratiche locali, e l'esperienza II desiderio di concorrere nella misura delle scarse mie forze al progresso, di un' arte nobilissima che sarà senpre la sorgente principale della nostra prosperità, mi coudusse ad intraprendere quest' ardua fatica, per la quale invoco per mezzo della S. V. Illustrissima l' assistenza ed il consiglio degli onorevoli miei Colleghi, che deputandomi a reg. gere la Scuola dell' Accademia mi offersero l' occeasione d'intrapreuderla. 
Tale, o signor Presidente, è il concelto che mi son fatlo dell' iusegnamento agrario, e tale il metodo adoperato per compartirlo nella mia scuola.

$\mathrm{Ma}$ esso sarebbe incompleto ove alla parle puramente scieutifica mancasse il riscontro della pratica, e l' evidenza dei fatti che acquista credito alle dottrine esposte nella scuola e serve a diffonderle fra i pratici coltivalori. II podere Mo. dello risponderà a questo uflicio importantissimo, e porrò ogni cura per dare ad esso un ordinaniento che possa soddisfare al bisoguo d' istruzione del maggior numero di proprietarii, dirigendone le colture in nodo imitabile a chi si trovi in analoghe condizioni, e mostrando col fatto che la Scienza e l'Arte possono darsi la mano per il riunoramento della nostra Agricoltura:

Sono con profondo rispetto.

Della S. V. Illustrissima

Pesaro 14 Giugno 1859 .

\section{Um̄o Devm̄o Obbm̄o seivo}

LUIGI GUIDI 


\section{A V VISO}

Col giorno 15 corrente si riaprirà la Scuola di Agricoltura di quest' Accademia nel solito locale del Palazzo Eleonori in via San Fraucesco di Paola num. 945 secondo piano.

11 Professure tratterrà uell' auno scolastico, terzo del corso.

1. Dell' Agricoltura speciale, esponendo le norme migliori per la coltivazione del Grano, Formentone ecc., Vite Olivo ecc, sia in ordinc all' arte in generale, che alle condizioni fisiche ed economiche del uostro territorio.

2. Della Pastorizic ossia dell' allevamento, governo e rendita del Bestiame grosso e nininuto, e come si possa migliorare le razze ed aumentarne il prodolto.

3. Della Contabilità Agricola, ossia del modo di rendersi conto dei titoli di entrata e spesa per tulti $\mathrm{i}$ raccolti $e$ industrie rurali e dedurne in fine la reudita netta.

Le Lezioni sono pubbliche, e ciascumo può intervenirvi come Uditore. Per concorrere al Premio e ricevere l' attestato conviene però essere ammesso come Scolare, pel che hasta far istanza al Deputato della Scuola, che è il chiarissimo signor marchese Pietro Petrucci.

Le lezioni si fanno nel Lunedi, Mercoledi e Sabbato di ciascuna settimana, e cominciano a mezzo giorno.

Per comodo poi di quei signori Possidenti e Fattori che non potessero seguire il corso triennale il Professore farà una classe a parte, in modo da compiere un corso elementarissimo uel giro dell' anuo, e per questi la Lezione comincierà allé I antimeridiane in ciascuno dei giorni sovraindicati.

Dato dalla Resideuza dell' Accademia questo di 8 No. vembre 1858 . 
(Allegato C)

Prodotlo della coltura della Lupinella sulla superficie di due quadrati di terreno pari ad ettari due.

I

La Lupinella veune seninata iusieme all'avena da falciare in verde sorra due quadrati di terreuo sabbioso-calcare, in eni nell' anuo precedeute era stato coltivato il formentoue, e che aveva ricevuto un buou rinuovo a vanga e 5 carri di concime di stalla all'Eutaro. La seminagione si eseguì vei primi di marzo, mediante una leggera erpicatura data sull' avena, la quale era stata seminata nel Novenibre precedente alla pari. o come dicesi a porconi, divisi da leggieri acquaj disposti in modo che la peudenza loro non superasse il $6 \%$.

La durata dei prati di lupinella è per media di auni 5 , dopo i quali conviene disfarli, tanto per la diminuzione grandissima del prodotto, quanto perchè torua più utile rimetterli nell' avicendamento, onde cavar profitto dalla fertilità accumulatasi nel suolo col lungo riposo, e gli avvanzi della vegetazione della Lupinella. Perciò il conto seguente uon presenta la vera parte proporzionale del beneficio di questa coltura, la quale al fiue dell' esperienza avrà dato una rendita molto maggiore. Iufatti dei tre anni che il medesino abbraccia, il primo è improduttivo, e sastiene tutte le spese dell' impianto, mentre al termine dell' intrapresa queste rimangono le stesse, e si aumenta invece il prodotto di altri due anni gravato soltanto dalle spese di aumninistrazioue e di raccolto.

Net conteggio vennero computate tulte quelle spese che realmente si sareblero incontrate in un fondo condotto direttamente dal padrone, essendosi determinato il valore delle giornate di lavoro degli uomini e degli aumah, secondo i prezzi correntị nel territorio.

Quanto agli alimenti per i Bovi e Bifolco, credo di non esserni discostato dal vero valutandone la spesa a baj. fo il giorno. 


\section{$-36-$}

Il prezzo dato al seme di Lupinella è quello che realmeute aveva in quell' auno.

Il valore del coneime fu pure desunto dai prezzi correnti secondo i quali fra noi un M.o Cubo di concime di stalla von fermentato vale circa baj. 6o, ed i carri del concime furono esaltamente misurati.

Nell' addebitare il costo del concime, tulti si accordano nel supporre che quando questo sia mezzanamente decomposto, metà venga consumato dalla coltura, cui viene dato direttamente, e melà ue resti nel suolo a beneficio delle successive collure, a debito delle quali vuol essere portato in modo che al termine dell' avvicendamento, o della speculazione, il costa di esso risulti perfettamente ammortizzato.

\section{S P E S E}

\section{ANNO PRIMO}

1. Aratura in settembre per preparare il terreno all' avena gionate N. 8, Bovi e Bifolco a baj. 90 l' una. . . Sc. $720-$

2. Giornate N. 16 di uomini per ripulire la terra e rompere le zolle, spunticchiare a baj. 15 » 2 40-

Somina Sc. 960

Netà dei quali a carico della Lupinella iı . . . . Sc. 480 -

3. Per toppi (0 di Lupinella col guscio a baj. 30 il toppo . . "12- -

4. Per spargere il seme giornate d'uomo N. 3 . " n-45-

5. ${ }^{\circ}$ Per erpicatura leggera giornata una e un tcrzo Buovie Bifolcon " 120

$6 .^{\circ}$ Canone del suolo valutato in perizia a Sc. 200 l' Ettare al :5

7. Interesse annuo della spesa al $b$ per cento . . . - \# 192 j

8. Interesse degli Sc. 750 conci- 
me non assorbito dalla coltura

\section{Riporto Sc. 40375}

precedente al 5 per cento " -37 !

Totale di spesa nel $10^{\circ}$ Anno — sic. $407 \%$ -

\section{ANNO SECONDO}

1. Giornate N. a d'uomoper restauro degli acquaj . : . Sc. $-30-$

$2 .^{\circ}$ Canone del suolo $20--$

$3 .^{\circ}$ Per fienatura e custodia del fieno giornate d' uomo $\mathrm{N} \cdot 22$.. " 330 -

4. ${ }^{\circ}$ Per trasporto del fieno giornate N. 2 Buoi e Bifolco.. " 180 -

5. Per rata annua ammortizzazione del concime in sorte Sc. 7 50 . . . " 187 :

$6{ }^{\circ}$ Interesse annuo al 5 per cento delle spese suddette . . . n 136 3

7. Interesse del concime non ammortizzato . . . " n-28-

Totale di spese nel 2. ${ }^{\circ}$ Anno —— Se. $\quad 38918$

\section{ANNO TERZO}

1. ${ }^{\circ}$ Giornate N. 4 d' uomo per lo spurgo degli acquaj … Sc. $-60 \div$

$2{ }^{\circ}$ Canone del suolo ..... n $20-$

3. Fienatura'e custodia del fieno giornate d'uomo N. 25 . n. $375-$

4. ${ }^{\circ}$ Trasporto del fieno giornate N. 3 buoi e Bifolco. . . $270-$

3.. Ammortizzazione del concime " $187 \mathrm{~B}$

$6^{\circ}$ Interesse al 5 per cento della somma suddetta . .,-18

Totale di spese nel $3 .^{\circ}$ Anno - Sc. 2910 5

Spesa totale nei tre Anni

Sc. $98 \quad 773$

\section{P R ODOT T I}

\section{ANNO SECONDO}

1. Fieno di primo taglio, pesato,

libb. 16,900 a Sc. 3.50 . Sc. $3915-$ 
2. Guajme, valutato, un quarto del

Riporto Sc. $5915-$

1. taglio ossia libb. 4000 fieno 14 :- Sc: $7313 \mathrm{~s}$

\section{ANNO TERZO}

1. Fieno di primo taglio pesato libb. 19,500: : Sc $6825-$

2. Guajme, valutato, a un quarto del $1^{\circ}$ taglio in libb. 4873 a 17062

Prodotto totale dei due Anni Sc. $138 \quad 462$

\section{BILANCIO}

PRODOTTO . Sc. 158.46 2

SPESE - . ¿ ^ 98.773

\section{Differenza attiva Sc. $\quad 59.689$}

$$
\text { N } 0 \text { 'T A }
$$

Essendosi differita fino ad oggi la stampa di questa relazione si è in grado di aggiungere il risultato definitivo dell' intrapresa, che si conchiuse col corrente anno, e che fu il seguente

\section{ANNO QUARTO}

1. Giornate N. 4 per lo spurgo degli acquaj : ... . Sc: 60 -

2. ${ }^{\circ}$ Canone del suolo

3. ${ }^{\circ}$ Fienatura e custodia del fieno gior. d'uomo N. 25 a baj. 15 n $375-$

4. Trasporto del fieno giornate 3 Buoi e Bifolco . . * 270 -

5. Ammortizzazione del Concime is 187 -

6. Interesse al 5 per cento delle spese precedenti :, , 148

7. Interesse del concime non ammortizzato . : $\quad 093$

Totale di spesa nel $40^{\circ}$ Anno $\longrightarrow$ Sc. $30 \quad 461$

\section{ANNO QUINTO}

1. Ripulitura degli acquaj giornate N. 6 a baj. 18 Sc. 108 -

2. Fienatura e custodia del fieno giornate N. 15 a baj. $18 \ldots$ 270- 
- Trasporto del fieno giornate 2

Riporto Sc. 378 - Sc. 30461

Buoi e Bifolco

$180-$

4. ${ }^{\circ}$ Ammortizzazione finale del concime

187 's

5. ${ }^{\circ}$ Canone del suolo . . . 20 -

6. ${ }^{\circ}$ Interesse al 5 per cento della somma suddetta . . " 1373

Totale di spesa del $3 .^{\circ}$ Anno - Sc. 28828

Spesa totale nel $4 .^{\circ}$ e 5.0 Anno Sc. $\quad 39289$

\section{P R O D O T T I}

\section{ANNO QUARTO}

1. Fieno dí primo taglio, pesato, libb. 17,500 a Sc. 3. 50 . Sc. $6123-$

2. Guaime valutato un quarto del primo taglio, libb. $4373 \ldots 15312$

Totale del prodotto — Sc. 76562

\section{ANNO QUINTO}

1.0 Fieno di primo taglio pesato libb. 10,000 a Sc 3.50 . Sc. $35-$

2. Guajme, valutato, un quarto del primo taglio

Totale del prodotto $\longrightarrow$ Sc. 4375

Prodotto totale dei due anni

Sc. 120312

\section{BILANCIO}

per $i$ cinque Anni

PRODOTTO $\quad \begin{array}{rll}\text { Sc. } 278774 \\ \text { SPESE }\end{array} \quad$ ». $15806 \quad 2$

Differenza attiva Sc. 120712

Diferenza attiva considerevole quanto si rifletta che durante tutti $\mathrm{i}$ cinque anni furono continuamente retribuiti $\mathrm{i}$ tre fattori della produzione stessa cioè $10^{\circ}$ il capitale del terreno; $2 .{ }^{\circ}$ il lavoro; $3 .{ }^{\circ}$ le scorte morte, e il capitale circolante in modo che questo costituisce l' utile effettivo di tale coltura; che ripartito per cinque anni e tenuto conto della media diferenza dei capitali attivi e passivi, quali realmente furono nel fondo durante questi cinque anni, la rendita media del capitale impiegato sarebbe stato di oltre Scudi $24 \%$ - 
(Allegato D)

\section{Drogrami PER I PREMi D' incoraggiamento.}

Quest'Accademia Agraria fin da parecchi anui rivolse i) pensiero a promuovere il miglioramento del Bestiame Buvino nel territorio della Provincia, al qual effetto nel 1853 distribui alcuni premi d'incoraggiamento ai Possessori degli animali riproduttori più perfetti. Ávendo l' esperienza dimostrato che da quell' epoca le razze del Bestiame grosso sono andate progressivamente migliorando per opportuna scelta di riproduttori e più sollecite cure di allevamento, si è stabilito di rinnovare in quest' anuo la premiazione, confidando che per tal modo col promuovere nei Possidenti l'emulazioue si potrà conseguire anche fra uoi quel perfezionamento delle razze dei Bestiami, che già in altri paesi venne oltenuto. Considerando però che l'allevamento del Bestiame minuto per la parte Montana della Provincia costituisce uua rendita cospicua, la quale può essere di molto aumentata col nighoramento delle razze medesime si volle che in questa circostauza la premiazione comprendesse tanto il Bestiame Bovino che le Pecore ed i Maiali.

Affiuchè dunque i Possidenti possano prepararsi per tempo a questa utile gara, fin d'ora si recano a cognizione loro le norme secondo le quali i Premi verrauno distribuiti.

1. La premiazione del Bestiame avrà luogo in questa Città nei giorni 1 t e 12 del venturo Settembre.

2. I Concorrenti al Premio dovrauno nella mattina del giorno i farsi inscrivere sul Registro a tal fine disposto nell' ufficio dell' Accademia, posto in casa Giglioni, al primo piano, via del Duomo N. civico 296.

3. Quattro Periti procederanno all' esame delle Bestie presentate al Concorso, e giudicheranuo in fatto del merito e qualità di esse.

4. Una Commissione composta di sei Membri, scelti nel seuo dell' Accademia, insieme ad un Veterinario presiederà al giudizio dei Periti, ed a norma di esso passerà ad asseguare i Premi. 
- 41 -

5. Se alcuno dei Periti nominati mancasse nei giorni asseguati, la Commissione provvederà a surrogarli cou altre persone capaci, dove nou reputi sufficiente il giudizio degli Intervenuti.

6, Non potranuo concorrere ai Premi, se non i Proprie. tari della Provincia.

7. I Premi da distribuirsi sono i seguenti.

I. ${ }^{\circ}$ Premio di Scudi 20 pel Toro, che per regolarità di forme meglio risponda all'ufficio di otlimo Riproduttore.

fra i rimanenti.

II. ${ }^{0}$ Premio di Scudi 5 pel Toro più distinto

III. ${ }^{\circ}$ Premio di Scudi r 5 per una Manza.

IV. ${ }^{\circ}$ Secondo Premio di Scudi 12 per altra Manza.

V. ${ }^{\circ}$ Due Premî di Scudi 10 ciascuno per due Vitelli dell' uno o dell'altro sesso che i Periti giudicheranno di forme più regolari, e meglio sviluppati in ordine alla loro età.

VI. ${ }^{\circ}$ Premio di Scudi 12 a chi presenterà complessivamente un Ariete con tre Agnelle, cha abbiauo migliori qualità di lana, ed attitudine all' ingrassamento.

VII. ${ }^{\circ}$ Premio di Scudi 6 per un Ariete.

VIII. ${ }^{\circ}$ Premio di Scudi xo per un Verro, che riunisca i caratteri piu rimarchevoli di robusta costituzione, e d'ingrassamento precoce.

come sopra.

IX.o Due premi di Scudi 5 l'uno per due Troje

8. Ciascun Premio sarà accompagnato da Diploma della Commissione, nel quale verà dichiarato it titolo pel quale fu conseguito.

9. Le condizioni alle quali dovranno soddisfare i Conrenti per aspirare ai Premi saranno le seguenti.

I. Il Proprietario dovrà provare, o con Certificato dell' Autorità Comunale, o con altro valido Documeuto, che i) Bestiame esposto al Concorso è allevato in questa Provincia.

2. $\mathrm{L}$ ctà dei Tori dovrà essere non minore di auni due, nè maggiore di cinque, e l'altezza non inferiore a palmi sei e mezzo di passetto romano archilettonico di once 12 , 08sia metri 1,452 . Ciascun Proprietario dovrà comprovare che il proprio Toro nei mesi di Aprile, Maggio, Giuguo, e Luglio dell' anuo corrente coperse in questa Provincia un au- 
mero di Vacche o Manze non minore di 3o, e per tal fine esibirà uu Registro esalto indicante il uome, cognome, contrada, e domicilio di quei proprietari, che abbiano portato le Manze, o Vacche a divenire feconde, il giorno e mese in cưi ciò ebbe luogo, e due testimani degni di fede dovrauno avere firmata, o crocesegnata ciascuna annotazione. Le Manze che si vorranuo esporre al Concorso, davranno essere nou minori di anui 3 , e di una altezza non inferiore a palmi 6 , ed once 4 di passetto romano architettonica ; cioè metri 1,485 . Il Proprietario dovrà giustificare che non abbiano mai figliało, e sieno state coperte in questa Provincia per la prima volta nei mesi opportuni dell' anno corrente.

I Vitelli e le Vitelle da qualunque Toro provenienti dovranuo avere tutti i denti da latte, e saranuo di altezza non inferiori di palmi cinque o mezzo di passetto architettonico, cioè metri $x, 229$. Si richiede inoltre che sieno senza difetto notabile specialmente nella cima e nelle gambe, e non abbiano la lingua bianca.

Finalmente il Proprietario dovrà comprovare che sono wati in questa Proviucia.

It Montone dovrà avere una età nou minore di 3 e nou maggiore di 5 auni; le Aguella dovranno avere passati i due auni, ma uon superati i tre. Il Proprietario dovrà provare validamente con attestato del Magistrato locale o altro idoneo documento, che il Montone ha coperto nella opportuna sta. gione un numero non minore di 3o Pecore. $L^{\circ}$ età assegnata per il Verro è quella non minore di due, e von maggiore di cinque anui. Per le Troie non minore di 18 mesi, e non maggiore di 6 anni. Il Possessore del Verro dovrà documentare che nella stagione opportuna esso ha coperto nel Territorio della Provincia 6 Scrofe.

Se i. Periti giudicassero che due individui sia del $\mathrm{Be}$ stiame grosso che del minuto fossero pari in perfezione, if premio verrà diviso fra i due Concorrenti.

Per i Vitelli e Manze di qualche pregio, che won conseguirono premio, la Commissione rilascerà un Certificato di menzione onorevole. Se il tempo impedisse che la premiazione si effettuasse nel gioruo stabilito, eșsa verrà trasportata nei due giorni successivi a quello in cui sarà trasferita la Fiera di S. Nicola, che cade il giorno dieci. 
Con apposito Manifesto al Pubblico verranuo accennate le premiazioni asseguate, e nominati insieme i Proprielari, e Coloni del Bestiame distinto.

Dato a Pesaro dalla Residenza dell' Accademia Agraria li 3 工 Marzo $\times 857$.

\section{IL PRESIDENTE}

\section{GIUSEPPE Arvocato LUGARESI}

L. GUIDI ff. di Segrelorio. 
Conforme al Programma pubblicato li 3r Marzo del corrente auno per la premiazione stabilita nello scopo di migliorare le razze dei Bestiami in questa Provincia il giorno I I del corrente Settembre ebbe luogo il relativo Concorso, e dopo esaurite le formalità prescritte nello stesso Programma, furono conferiti i soli Premî qui àppresso descritti.

Premio di Scudi 20 per un Toro di proprietà della Eccellentissima Casa CASTELBARCO-ALBANI, ritenuto dal Colono Ridolfi Giuseppe.

Premio di Scudi r 5 per altro Toro di propriełà del. l' Eminentissimo signor Cardinale LUIGI CIACCHI ritenuto dal Colono Raisini Raffaele.

Certificati di Onorevole Menzione di lode.

I. Per un Vitello di proprietà dell' Eccellentissima Casa CASTELBARCO-ALBANI ritenuto dal Colono $\mathrm{Pa}$ gnini Luigi.

2. Per una Vitella di proprietà de! Veuerabile Ospedale di SAN SALVATORE ritenuta dal Colono Mazzanti Antonio.

3. Per una Vitella di proprietà del signor Conte GORDIANO PERTICARI riteunta dal Colono Grommetti Raffaele.

4. Per un verro di proprietà dell' Eccellentissima Casa CASTELBARCO-ALBANI ritenuto dal Colowo $M a$ tioli Antonio.

5. Por una Troja di proprielà del signor ANDREA MARZETTI ritenuta dal Colono Sante Marchionni.

6. Per un' Ariete di proprietà del sig. Conte FRANCESCO BRACCI.VATIELLI ritenuto dal Colono $D^{\prime} A n$ geli Antonio. 


\title{
$-45-$
}

7. Per tre Peeore di proprietà del signor Conte VIN. CENZO MACHIRELLI, ritenute dal Colono Sardini Ma. riano.

Dalla Residenza dell' accademia Agraria in Pesaro li 14 Seltembre 1857 .

\section{IL PRESIDENTE ATUSEPRE AVv. HUGAIEST}

\author{
Il ff. di Segretario \\ LUIGI GUIDI
}


Avendo l' Accademia Agraria stabilito di distribuire anche in quest' anno i consueti premj alla fine di promuovere il miglioramento delle razze dei Bestiami nel territorio della Provincia, si recano a cognizione dei Possidenti le norme secondo le quali i premj suddetti verrauno conferiti.

1. La premiazione del Bestiame avrà luogo in Urbino nel gioruo 3r del prossimo Agosto. Sono ammessi al Concorso i soli Possidenti della Provincia i quali dovranuo inscriversi nel giorno 29 e 3 o sul registro a tal fime disposto nel Palazzo Comunale della Città stessa.

2. Sei Periti, due del territorio di Urbino, due del Monte-Feltro, due di Pesaro procederanno all' esame delle Bestie presentate al Concorso, e giudicherauno del merito assoluto di esse.

3. Una Commissione composta di sei Membri scelti fra i Socj ordinarj dell' Accademia insieme al Veterinario della Citlà di Urbino presiederà al giudizio dei Periti, ed a norma di esso asseguerà $i$ premj.

4. Mancando alcuno dei Periti, la Commissioue provvederầ a surrogarlo, dove non reputi sufficiente il numero degli intervenuti.

5. I premj da distribuirsi sono $i$ seguenti :

1. Premio di Scudi 20 al Toro che per regolarità ed amplitudine di forme meglio risponda all' ufficio di ottimo riprodultore per le razze di pianura.

II. Altro premio eguale di Scudi 20 pel Toro, che riunisca i caratteri più importanti di buon riproduttore per le razze di monte.

III. Due premj di seudi 5 l' uno, per due Manze, l' una delle quali riunisca i maggiori pregi delle razze di piano, l'altra la leggerezza rusticità ecc. delile razze di monte.

IV. Due premj di scudi* yo ciascuno, per due Vitelli dell' uno e dell' altro sesso che i Periti giudicherauno di forme più regolari e meglio sviluppati riguardo l' età e la razza loro. 
V. Premio complessivo di scudi 12 per un Montone e tre Agnelle le quali presentino migliori qualitå di laua, regolarità di forme ed attitudiue all' ingrassamento.

VI. Premio di scudi 6 per un Montone solo.

VII. Tre premii di Scudi 2 l' uno a ehi presenti due Aguelli dell' anno me lio sviluppati e nutriti.

VIII. Premio di Scudi io per un Verro che riunisca caratteri piü rimarchevoli, di robusta costituzione e d'ingrassamento precoce. sopra.

IX. Due premii di Scudi 5 l' uno per due Troje, come

6. Ciascun premio sarà accompagnato da Diploma dell'Accademia, nel quale verrà diehiarato il titolo per cui fu conseguito.

7. Le condizioni alle quali dovranno soddisfare i Concorrenti per aspirare al prenio sono le seguenti.

I. Che il P'roprietario provi o con certificato dell'Autorità Comunale o con altro valido documeuto che it Bestiame esposto al concorso è nato nella Provincia, o almeno da un anno vi è stato introdotto.

II. Che i.Tori abbiano superata l' età di due anni, ma non oltrepassata quella di cincque, ed abbiano coperto un numero di 36 Vacche, al qual fiue li Esponenti esibiranno un registro indicante il nome, cognome e domicilio dei Proprietarii delle medesime. Sarebbe anzi desiderabile che il numero di queste non oltrepassasse le 40, onde impedire l' indebolimento del maschio, e le cattive couseguenze che ne risente la prole.

III. Che le Manze sieno di età uou minore di anni tre, non abbiano mai figliato, e siano munite di un documento che comprovi che per la prima volta venuero coperte da un Toro della Provincia nei mesi opportuni dell' anno corrente.

IV. Che i Vitelli e le Vitelle da qualunque Toro provenienti, siano nati nella Provincia, cosa che il Proprietario dovrà documentare con idoneo certificato.

V. Che il Montone abbia compiuto i tre anni, ma non oltrepassati i cinque, e le Agnelle superate $i$ due, ma von oltrepassati i tre anni, e quanto al Montone che risulti da un documento aver esso nella stagione opportuna coperto un numero di 30 pecore. 
VI. Che il Verro abbia compiuti i due anui, ma non superati $i$ cinque. $L$ ' età assegnata per le Troje è compresa fra li 18 mesi e li 6 auui. Il possessore del Verro dovrà do. cumentare che nella stagioue opportuna esso ha coperto 6 Scrofe nella Provincia.

Se i Periti gindicassero che due animali fossern pari in perfezione, il premio verrà estratto a sorte.

Per i Vitelli e le Manze di qualche pregio che non avessero conseguito premio, la Commissione rilascerà un certifi. cato di menzione onorevole.

Se il tempo impedisse la premiazioue nel giorno stabilito, essa verrà trasportata al giorno suecessivo.

Con apposito manifeslo verranuo accennate le premiazioni asseguate, e nominati i Proprietarii e Coloni del Bestiame distinto

Dato a Pesaro dalla Residenza dell' Accademia questo. di r Luglio I 858.

\section{IL PRESIDENTE \\ GHUSERPR Avv. HUGAIRES}

LUIGI GUIDI ff. di Segrelario, 


\section{ACCADEVIA DI AGRICOLTURA IN PESARO}

Conforme al Programma pubblicato il r. Luglio del corrente Auno per la premiazione stabilita al fine di migliorare le razze dei Bestiani nella provincia, il giorno 3 I Agosto ebbe luogo in Urbino il relativo concorso, e dopo esanrite le formalità prescritte nello stesso Programma in presenza dell' Illustrissima Magistratura della Città corlesemente intervenuta, furono conferiti i Premii qui appresso descritti.

I. Premio di Scudi 20 per un Toro delle razze di Piano preseutato dal signor ROMOALDO MERCURI di S. Angelo in Vado, ritenuto dal Colono Giovanni Matteucci.

II. Premio di scudi 20 per un Torn delle razze di Monte presentato dal signor Marchese LUIGI BARBI di Gubbio.

III. Premio di scudi i 5 per una Manza presentata dal MONASTERO DELLE BENEDETTINE di Urbino, e rilenuta dal Colono Crescentina Pari.

IV. Prensio di scudi ro per una Vitella di latte no. strana presentata dal signor GIROLA MO PICCWNI di Fermiguano, e riteuuta dal Colono Sante Pascucci

V. Premio di scudi io per una Vltella Mongana presentata dal siguor Conte BERNARDINO CASTRACANE. di Urbino. e ritenuta dal Colono Crescentino Lucci

VI. Premio di scudi 8 per un Montone presentato dal signor Conte BERNARDINo CASTRACAXE di Untho, c ritenuta dal Colono C'rescentino Lucci.

VII. Premio di scudi 4 per una Pecora presentata dat signor Conle TITO PALMA di Urbino, e riteuta dal Cólono Pietro Angelini.

VIII. Premio di soudi ro per un Verro presentato dal signor Conte BERNARDINo CASTRACANE di Urbinn. e ritenuto dal Colouo Pietro Carloni.

IX. Prensio di scudi 5 per una Troja presentata dal 
signor LUIGI MAZZA di Urbino, e ritenuta dal Colono Pietro Angeli.

$\mathbf{X}$. Premio di scudi 5 per: una Troja presentata dal siguor ANTONIO PERGAMI di Urbino, e ritenuta dal Colono Giovanni Donnini.

Si rilasciarono inoltre i seguenti Certificati di onorevole meuzione di lode.

1. Al signor Conte TITO PALMA di Urbino per un Toro della razza di Piano ritenuto dal Colono Pietro Ruggeri.

2. Al signor Marchese LUIGI BARBI di Gubbio per una Manza della razza di Monte.

Dalla residenza dell' Accademia Agraria in Pesaro li 2 Settembre 1858.

\section{IL PRFSIDENTE \\ GIUSEPPE Avvocato LUGARESI}

Il ff. di Segretario

LUIGI GUIDI

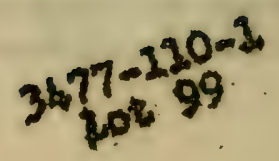








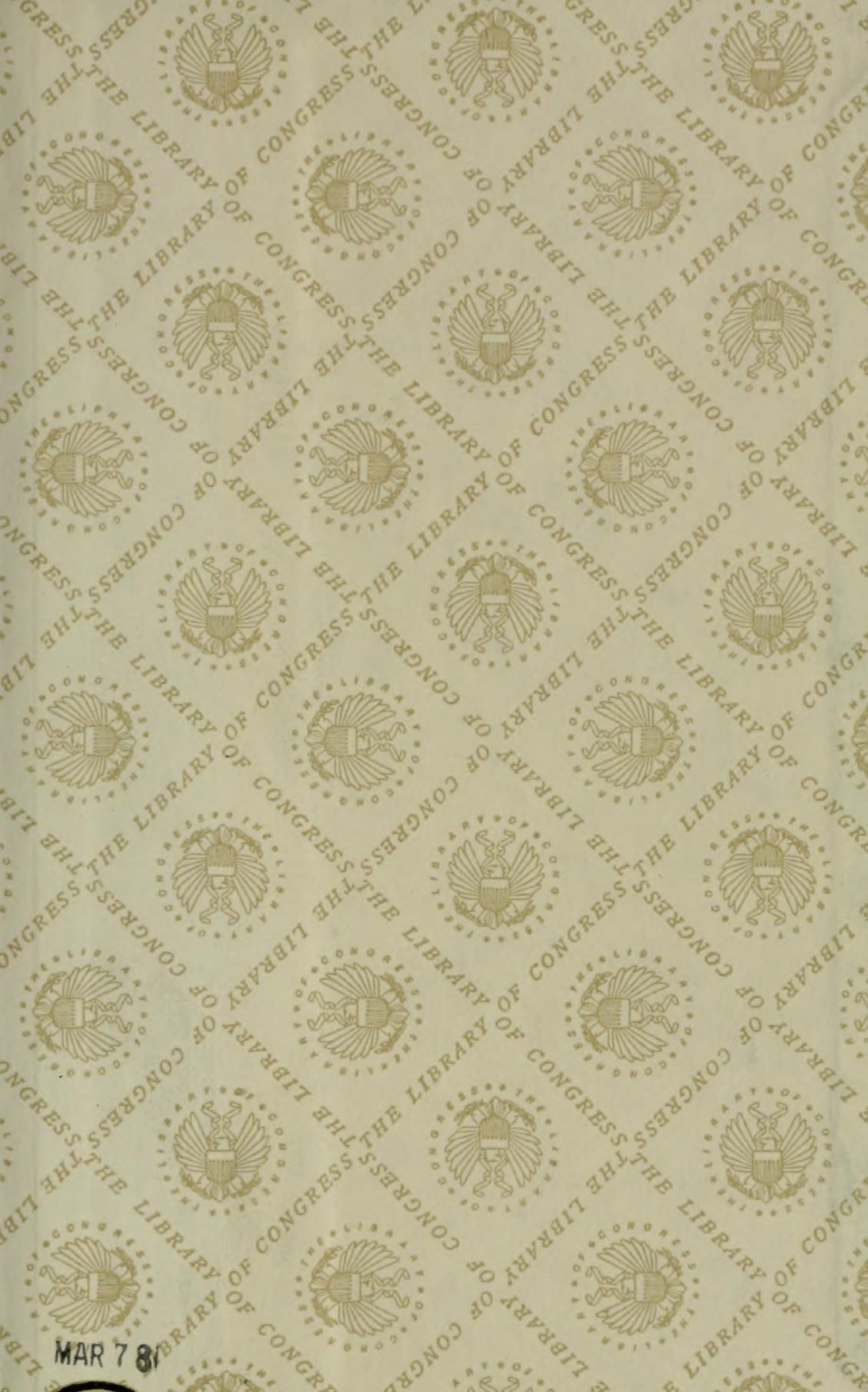




\section{LIBRARY OF CONGRESS}

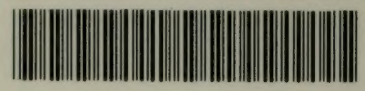

00026001092 